Article

\title{
Three Melanin Pathway Genes, TH, yellow, and aaNAT, Regulate Pigmentation in the Twin-Spotted Assassin Bug, Platymeris biguttatus (Linnaeus)
}

\author{
Yinqiao Zhang ${ }^{\circledR}, \mathrm{Hu} \mathrm{Li} @$, Juan Du, Junzheng Zhang, Jie Shen *® and Wanzhi Cai * \\ Department of Entomology and MOA Key Lab of Pest Monitoring and Green Management, College of Plant \\ Protection, China Agricultural University, Beijing 100193, China; zhangyinqiao519@126.com (Y.Z.); \\ tigerleecau@hotmail.com (H.L.); dujuan9981@163.com (J.D.); 2015008@cau.edu.cn (J.Z.) \\ * Correspondence: shenjie@cau.edu.cn (J.S.); caiwz@cau.edu.cn (W.C.)
}

Received: 28 April 2019; Accepted: 25 May 2019; Published: 3 June 2019

\begin{abstract}
Pigmentation plays a vital role in insect survival and reproduction. Many melanin pathway genes have been studied in holometabolous insects; however, they have only been studied in two hemimetabolous insect genera, Oncopeltus and Periplaneta. Here we analyzed three melanin pathway genes (TH, yellow, and aaNAT) using RNA interference (RNAi) in another hemimetabolous insect, namely the twin-spotted assassin bug, Platymeris biguttatus. TH was highly expressed in freshly molted nymphs and adults. TH RNAi resulted in a complete loss of black pigment, with yellow coloration maintained. Therefore, black pigment in this assassin bug is solely generated from the melanin pathway, whereas yellow pigment is generated from other unknown pigmentation pathways. yellow and aaNAT were highly expressed in the white spot of the hemelytra. Downregulation of yellow caused a brown phenotype with high mortality, indicating an important role of yellow functions in cuticle formation and in the process of converting melanin from brown to black. Interestingly, aaNAT RNAi caused not only loss of white pigment, but also loss of yellow and red pigments. This phenotype of aaNAT has not been reported in other insects. Our results provide new information for understanding the melanin pathway in which aaNAT is essential for the formation of colorless patterns.
\end{abstract}

Keywords: Platymeris biguttatus; pigmentation; tyrosine hydroxylase (TH); yellow; arylalkylamine- $N$-acetyltransferase (aaNAT)

\section{Introduction}

Pigmentation is one of the most distinctive and variable features of insects. Pigmentation differs substantially between insect species, populations, and individuals, and even in different regions of the body [1,2]. Pigmentation also plays indispensable roles in behavioral, physiological, and reproductive processes [3]. Most genetic and molecular mechanisms controlling pigmentation have been elucidated in the model species Drosophila melanogaster [4-6]. Pigment synthesis begins with the conversion of tyrosine to dihydroxyphenylalanine (DOPA) by tyrosine hydroxylase, which is encoded by the TH gene. Some DOPA molecules are then converted to DOPA melanin (black), and the yellow-family genes play important roles in this process. DOPA can also be converted to dopamine by DOPA decarboxylase (Ddc), after which dopamine can go through three metabolic branches. The first branch involves the direct production of dopamine melanin (brown/black). The second branch involves conversion to $N$ - $\beta$-alanyl dopamine (NBAD) by ebony, which generates yellow pigment, and can be reversed by tan. The last branch involves the production of N-acetyl dopamine (NADA), which is the precursor 
of colorless sclerotin, and this process depends on the activity of arylalkylamine- $N$-acetyltransferase $(a a N A T)$. These melanin precursors are synthesized and secreted from the imaginal epidermis and then incorporated into the cuticle [7-9].

Functional analyses in Drosophila first shed light on basic information about genes that regulate pigmentation, such as Ddc, yellow, tan, and ebony [6,8,10-12]. Pigmentation has also been studied in other groups of insects. For example, Tribolium castaneum serves as another influential insect model, and its melanin genes (TH, Ddc, yellow, and ebony) have been studied [13-17]. In addition, pigmentation studies have been conducted in some other holometabolous insects, such as in Heliconius, Papilio, Monochamus, Apis, and Aedes genera [18-24]. Since 2010, the role of aaNAT in pigmentation was functionally analyzed in Bombyx mori, and it was found that RNAi against aaNAT caused a partial or complete black phenotype in adults [25-27]. In Tribolium, aaNAT was not required for pigmentation of non-adult cuticles, although it played critical roles in the morphology and pigmentation of adult cuticles [28]. To date, systematic research of these melanin genes has been performed only in two hemimetabolous insects, Oncopeltus and Periplaneta [29-31]. RNAi against aaNAT in these two hemimetabolous insects caused region-specific phenotypes. In Oncopeltus, only the anal lobe region of the hindwing became melanized in aaNAT RNAi adults. In Periplaneta, only the colorless region of the pronotum became melanized in dsaaNAT-treated adults [30]. Therefore, the genes involved in pigmentation are functionally diverse in these two types of metamorphosis in insects. Naturally, questions arise, such as whether all melanin genes work differently in holometabolous and hemimetabolous insects, or whether melanin genes function similarly among all hemimetabolous insects. Therefore, it is necessary to perform functional analyses of the melanin pathway in other hemimetabolous insects.

Platymeris biguttatus, with brightly contrasting colors, is very suitable for performing pigmentation studies. It is mostly black throughout the body with a yellow annulation on the leg and a white spot on the hemelytra. In addition, this insect has a body length of approximately $32 \mathrm{~mm}$, and shows high efficiency of double-stranded RNA (dsRNA) injection. The expression profiles of three essential genes in the melanin pathway, $T H$, yellow, and aaNAT, were analyzed along with developmental stages, pigmentation time points, and specific tissues with respect to color patterns. The functions of these genes were assayed by RNAi. Our results suggest that the black pigmentation was solely generated from the melanin pathway in this assassin bug. In addition, downregulation of yellow caused a brown phenotype with high mortality. Interestingly, aaNAT RNAi induced completely black pigmentation, with the loss of all other color patterns.

\section{Results}

\subsection{Expression Patterns of Three Melanin Pathway Genes (TH, yellow, and aaNAT)}

Before using the RNAi method to analyze the functions of $T H$, yellow, and aaNAT in the color formation of this assassin bug, it was necessary to determine their expression patterns. We performed quantitative real-time PCR of these three genes at different developmental stages, different pigmentation time points, and body regions with different colors (Figure 1). The expression level of $T H$ at different developmental stages was fluctuant. It was significantly lower in the first-instar nymphs than in others (Figure 1A). During the pigmentation process, the highest expression level of $T H$ was detected in the freshly molted fifth-instar nymphs or adults, and gradually decreased until the pigmentation was completed (Figure 1B). TH expression level was significantly higher in the black and white regions of adults than in other regions (Figure 1C). The expression level of yellow gradually increased from the firstto the fifth-instar nymphs, but significantly declined in adults (Figure 1D). In contrast, the expression level of aaNAT in adults was significantly higher than that in nymphs (Figure 1G). Similar to TH, expression levels of yellow and aaNAT were significantly higher in the $0 \mathrm{~h}$ nymphs or adults than those in the $48 \mathrm{~h}$ nymphs or adults (Figure 1E,H). During the pigmentation process, the expression level of yellow was significantly increased in the $5 \mathrm{~h}$ adults and $10 \mathrm{~h}$ adults (Figure $1 \mathrm{E}$ ), while the expression level of aaNAT was the highest in the $10 \mathrm{~h}$ adults (Figure $1 \mathrm{H})$. Interestingly, yellow expression level in 
the white spot of the hemelytra was as high as in the black regions of adults (Figure 1F). The expression level of $a a N A T$ was significantly higher in the white spot than in other regions (Figure 1I).
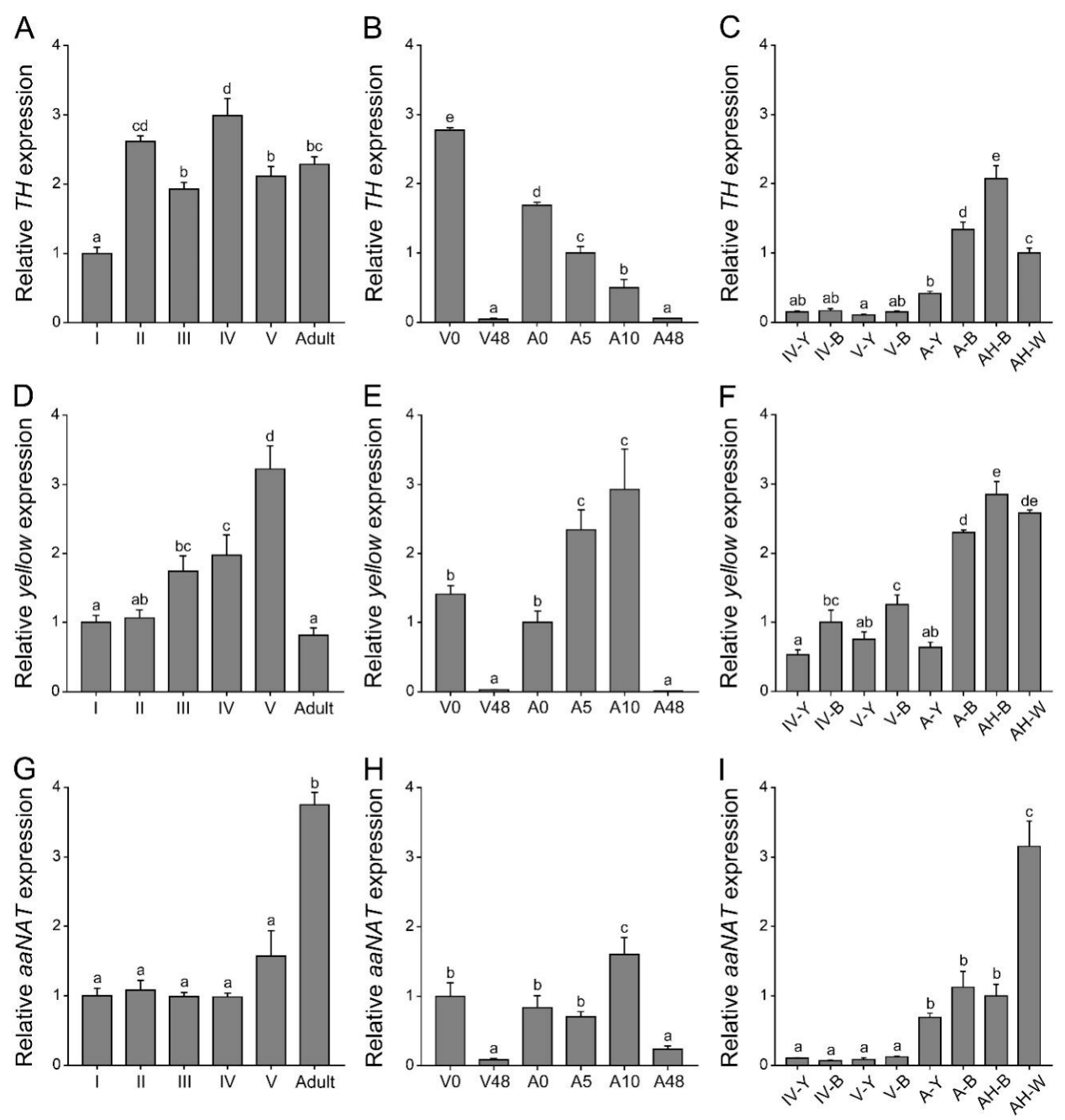

Figure 1. Expression profiles of $T H$, yellow, and aaNAT in P. biguttatus. (A,D,G) Relative expression levels of $T H$, yellow, and aaNAT were detected by quantitative real-time PCR at different developmental stages. All these nymphs and adults have completed the pigmentation process before RNA extraction. $(\mathbf{B}, \mathbf{E}, \mathbf{H})$ Relative expression levels of these three genes at different pigmentation time points. (C,F,I) Relative expression levels of these three genes in body regions with different color patterns from different developmental stages. I-V, the first- to fifth-instar nymphs; A, adults; $0,5,10$, and $48 \mathrm{~h}$ of the corresponding instar nymphs; $\mathrm{Y}$, the yellow annulation of the leg; $\mathrm{B}$, the black region of the leg; $\mathrm{H}-\mathrm{B}$, the black region of the hemelytra; $\mathrm{H}-\mathrm{W}$, the white spot of the hemelytra. Expression levels were normalized to the expression of EF1 $\alpha$ of P. biguttatus. Data are shown as the mean values \pm SE (error bars) $(n=3)$. Different letters $(a, b, c, d$, and e) on the error bars indicate statistically significant differences (one-way ANOVA analysis, $p<0.05)$.

Significant differences in the temporal and spatial expression profiles were noted among these three genes. RNAi experiments are needed to determine what roles they play during the pigmentation process in this assassin bug and what, if any, relationships occur between them.

\subsection{Functional Analyses of $\mathrm{TH}$ and yellow}

The freshly molted fifth-instar nymph or adult was bright red, gradually turned brown after about five hours (Figures S1B and S2B), then turned dark brown after about ten hours (Figure S2C), and finally 
turned black after the pigmentation progress was completed (Figures S1C and S2D). This process took $18 \mathrm{~h}$ and $48 \mathrm{~h}$ for the fifth-instar nymph and the adult, respectively. The fully pigmented fifth-instar nymph was mostly black, with a yellow annulation on the leg (Figure S1C). The abdomen changed from black to brownish red after feeding (Figure S1D). Similarly, the body of the adult was mostly black, with a yellow annulation on the leg and a white spot on the hemelytra (Figure S2D). The region of the dorsal abdomen covered by the forewings was red (Figure S2E). The membranous hindwing was light brown with a fenestrate area (Figure S2F, the black arrowhead). To explore the role of the melanin pathway in the pigmentation of P. biguttatus, we selected three essential melanin genes: $T H$, yellow, and aaNAT to perform RNAi experiments based on the color pattern of this assassin bug. The phylogenetic analysis confirmed that $T H$, yellow, and aaNAT represent orthologs of this assassin bug (Figure S3). Then, we performed quantitative real-time PCR analysis on each gene to test the efficiency of the RNAi experiments, and the results showed that the transcription levels of these genes were significantly lower in the dsRNA-treated groups than in the dsGFP-treated groups (Figure S4). Therefore, the observed phenotype was caused by the decrease in the expression level of the corresponding gene.

First, we needed to determine whether the black pigment of this species was synthesized by the melanin pathway. Since TH catalyzes the initial step of this pathway, we injected $20 \mu \mathrm{g}$ dsTH into each of the early fourth-instar nymphs (about seven days old). The same amount of dsGFP was injected into the nymph of the same developmental stage as a control. In total, of the 11 dsGFP nymphs, three were used to extract RNA, seven molted into the fifth instar, and one died 15 days after the injection (in the middle instar). Nymphs treated with dsGFP molted into the fifth instar with a normal pigmentation pattern (Figure 2A). Of the 19 nymphs injected with dsTH, three were used to extract RNA, only four of them successfully molted to the next instar, and 12 died during the molting process. The four fifth-instar nymphs also died within seven days and were disabled in feeding. RNAi of TH led to the loss of black pigment throughout the body, but yellow pigment was not affected (Figure 2E), and the effects were consistent among these four fifth-instar nymphs. Some bristles were light brown or even white, especially on the head and tibia of the dsTH nymphs (Figure 2F,G). However, there were still some dark brown markings on the cuticle (Figure 2E,H). Down regulation of $T H$ also caused defects in cuticle sclerotization. This resulted in warped legs (Figure 2E), deformed compound eyes (Figure 2F), and wrinkled wing discs (Figure 2H). The bristles of dsGFP-treated nymphs were long and straight (Figure 2B,C), but a lot of the bristles of the dsTH-treated nymphs were short and limp (Figure 2F,G). Moreover, the cuticle of the dsTH nymphs was less granulose and smoother than that of the control nymphs.

The yellow gene can promote the cuticular melanization process. To determine its function in this assassin bug, we performed yellow RNAi experiments. Of the 21 nymphs injected with dsyellow, three were used to extract RNA, 16 of them died as fourth-instar nymphs during the expected molting period, and their body walls were very soft before they died, only two successfully molted to fifth-instar nymphs, and they died in the early instar. These two yellow RNAi nymphs showed a brown phenotype, with the lack of black pigment (Figure 3E). Loss of yellow function did not affect the normal development of cuticles (Figure 3F,H) or bristles (Figure 3G). 
A

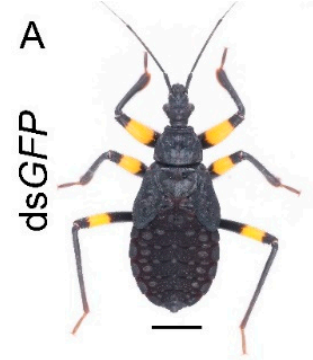

E

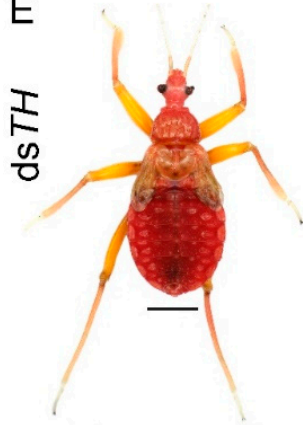

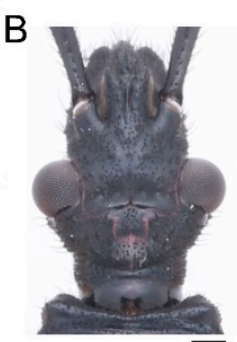

$\mathrm{F}$

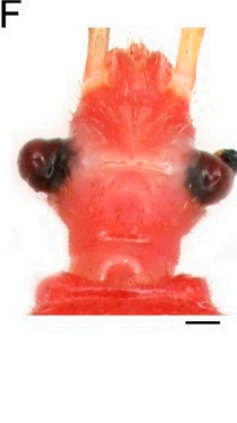

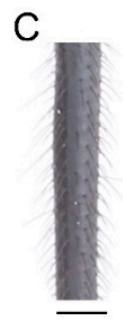

G

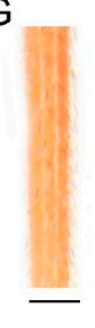

D

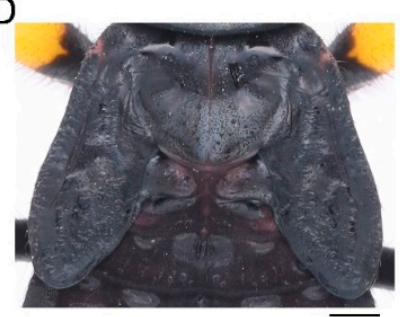

$\mathrm{H}$

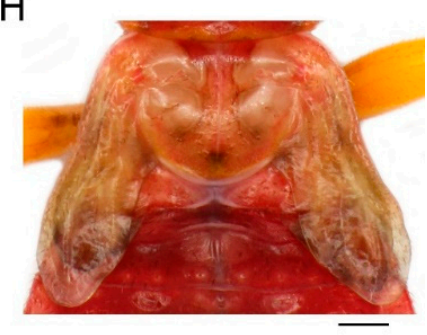

Figure 2. Functions of $T H$ in P. biguttatus nymphs. Nymphs treated with dsGFP showed normal pigmentation patterns (A), well-developed cuticles (B,D), and bristles (C). The dsTH nymphs displayed nearly a complete loss of black pigment, with yellow pigment not affected (E). The deformed compound eyes and white bristles on the head (F). The short and limp bristles on the tibia (G). The wrinkled wing discs and some dark brown markings on the cuticle (H). Scale bars, $5 \mathrm{~mm}(\mathbf{A}, \mathbf{E}), 500 \mu \mathrm{m}(\mathbf{B}, \mathbf{F}), 250 \mu \mathrm{m}$ $(\mathbf{C}, \mathrm{G}), 1.25 \mathrm{~mm}(\mathbf{D}, \mathrm{H})$.
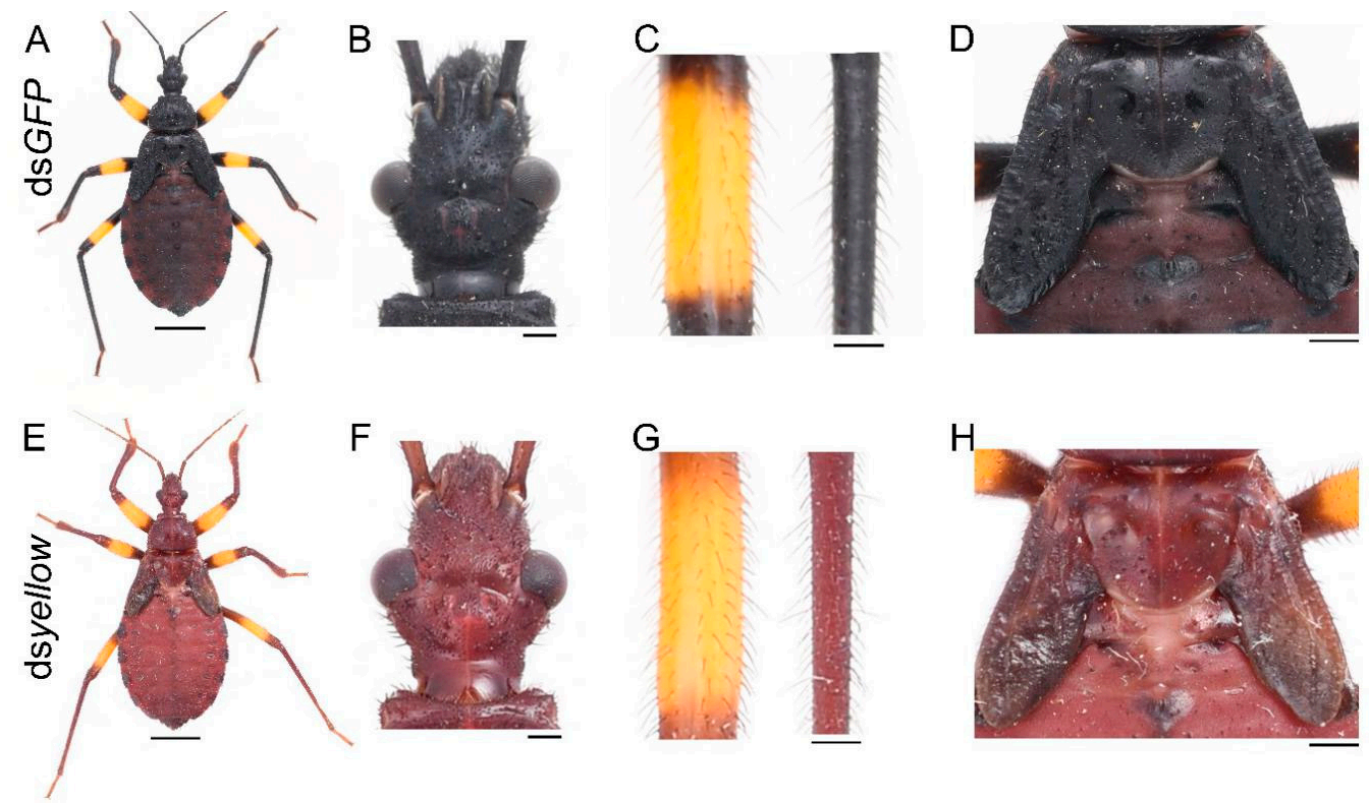

Figure 3. Functions of yellow in P. biguttatus nymphs. Nymphs treated with dsGFP showed normal pigmentation patterns (A), well-developed cuticles (B,D) and bristles (C). Nymphs injected with dsyellow showed a brown phenotype (E), with intact cuticles $(\mathbf{F}, \mathbf{H})$ and bristles $(\mathbf{G})$. Scale bars, $5 \mathrm{~mm}$ $(\mathbf{A}, \mathbf{E}), 500 \mu \mathrm{m}(\mathbf{B}, \mathbf{F}), 250 \mu \mathrm{m}(\mathbf{C}, \mathrm{G}), 1.25 \mathrm{~mm}(\mathbf{D}, \mathbf{H})$.

\subsection{Functional Analyses of aaNAT}

To investigate whether aaNAT was responsible for the formation of the white spot of the hemelytra, we performed aaNAT RNAi in the early fourth-instar nymphs. Of the 21 injected fourth-instar nymphs, no nymphs died, three were used to extract RNA, and 18 molted to fifth-instar nymphs. Then, 
we injected $20 \mu \mathrm{g}$ dsaaNAT into each of the 18 fifth-instar nymphs (about seven days old), four of them were used to extract RNA, seven molted to adults, and seven died due to aborted ecdysis. Interestingly, RNAi against the aaNAT gene generated a distinct pigmentation pattern. The body colors of the fifth-instar nymphs treated with dsaaNAT were darker than that of those treated with dsGFP (Figure 4G-I,K,L). Moreover, the yellow annulation of the leg turned black after aaNAT RNAi (Figure 4G,H,L). However, the color of the sternum was not apparently changed (Figure 4J). A global black phenotype was observed in all adults treated with dsaaNAT. The expansion of black pigment was observed throughout the entire body regions, including the brownish red ocelli on the head (Figure 5H, the white arrowhead), the white spot on the hemelytra (Figure 5I), the yellow annulation on the leg (Figure 5J), the red dorsal abdomen (Figure 5K), and the white fenestrate area on the hindwing (Figure 5L). RNAi for aaNAT had no effect on the development of cuticles in nymphs or adults (Figures 4 and 5).
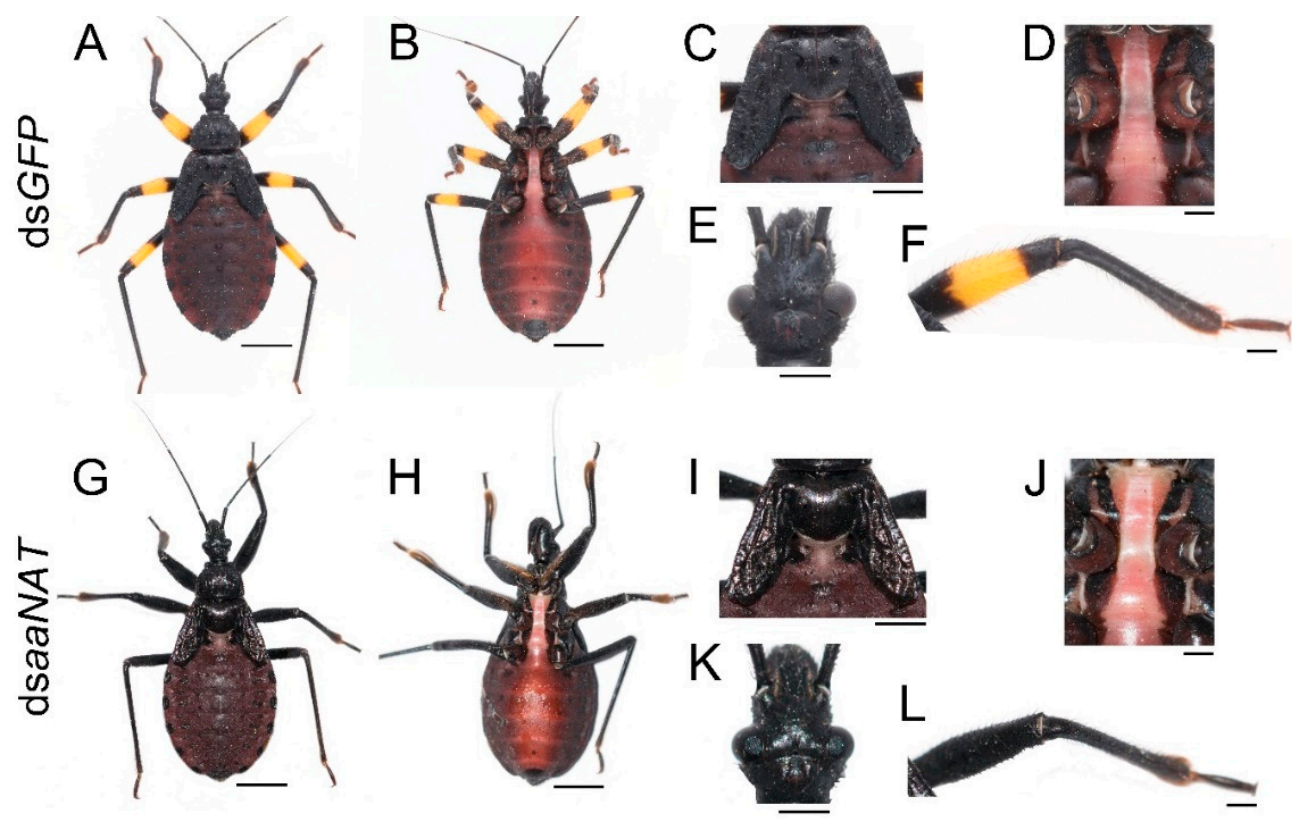

Figure 4. Functions of aaNAT in P. biguttatus nymphs. Nymphs treated with dsGFP showed normal pigmentation patterns of the dorsal (A) and ventral side (B), including the wing discs (C), the sternum (D), the head (E), and the leg (F). Nymphs treated with dsaaNAT showed darker pigmentation patterns than those treated with dsGFP $(\mathbf{G}-\mathbf{I}, \mathbf{K})$, the yellow annulation of the leg turned black $(\mathbf{L})$, while the color of sternum was not apparently changed (J). Scale bars, $5 \mathrm{~mm}(\mathbf{A}, \mathbf{B}, \mathbf{G}, \mathbf{H}), 2.5 \mathrm{~mm}(\mathbf{C}, \mathbf{I}), 1.25 \mathrm{~mm}$ $(\mathbf{E}, \mathbf{K}), 1 \mathrm{~mm}(\mathbf{D}, \mathbf{F}, \mathbf{J}, \mathbf{L})$. 

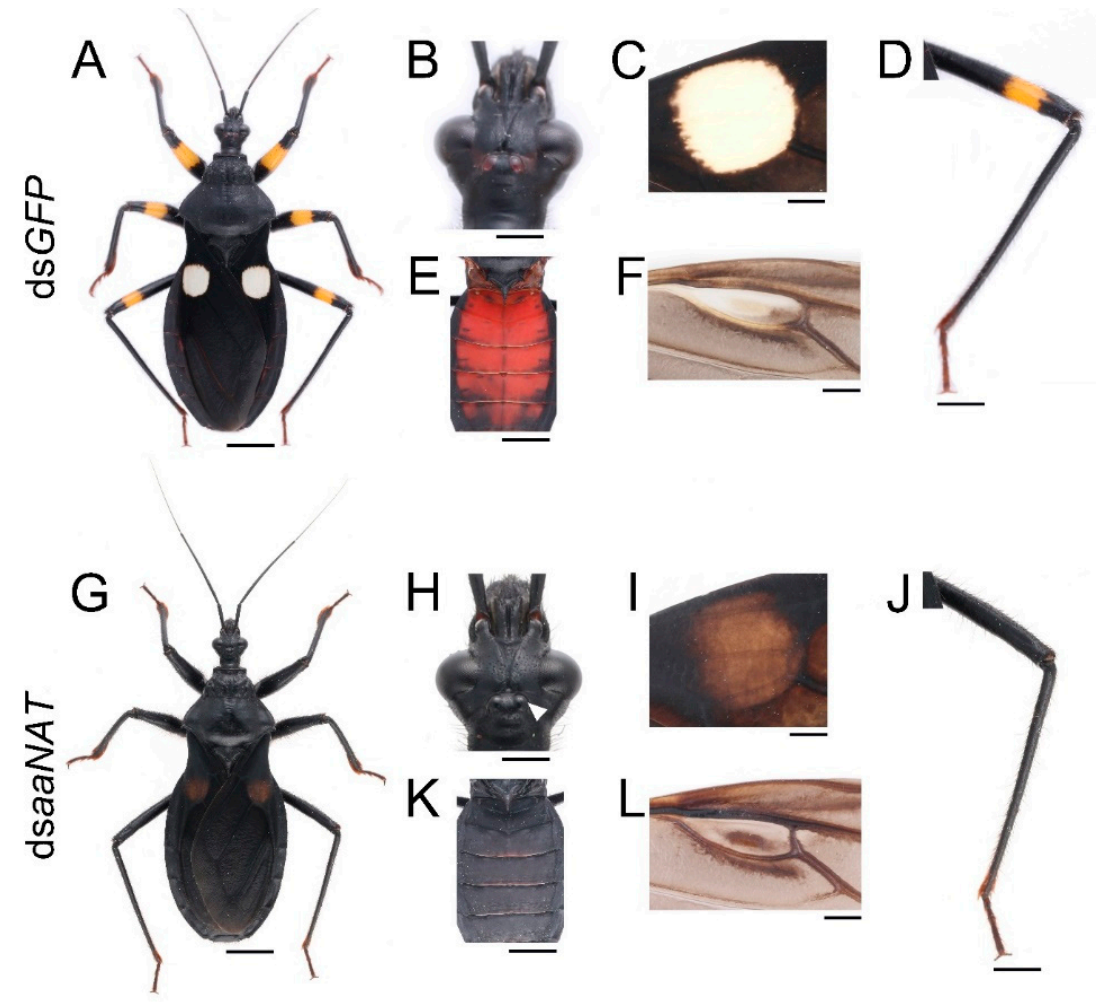

Figure 5. Functions of aaNAT in P. biguttatus adults. Adults treated with dsGFP showed normal pigmentation patterns (A), including the brownish red of ocelli (B), the white of the hemelytra (C), the yellow of the annulation on the leg (D), the red of the dorsal abdomen (E), and the white of the fenestrate area on the hindwing (F). (G) Adults treated with dsaaNAT showed the expansion of black pigment in the head $(\mathbf{H})$, the forewing and the hindwing $(\mathbf{I}, \mathbf{L})$, the leg $(\mathbf{J})$, and the dorsal abdomen $(\mathbf{K})$. The white arrowhead $(\mathbf{H})$ indicates the ocelli. Scale bars, $5 \mathrm{~mm}(\mathbf{A}, \mathbf{E}, \mathbf{G}, \mathbf{K}), 2.5 \mathrm{~mm}(\mathbf{D}, \mathbf{J}), 1.25 \mathrm{~mm}(\mathbf{B}, \mathbf{H})$, $1 \mathrm{~mm}(\mathbf{C}, \mathbf{F}, \mathbf{I}, \mathbf{L})$.

\section{Discussion}

\subsection{Black Pigment Is Solely Generated by the Melanin Pathway}

In this study, we report the first application of RNAi in this assassin bug. RNAi has been proven to be an essential tool for studying the functions of genes in non-model insects, for which genetic tools are lacking [32]. However, the efficiency of RNAi varies greatly among different insect species [33]. Among hemipteran insects, RNAi performed poorly in the pea aphid, Acyrthosiphon pisum, while it was highly effective in the milkweed bug, Oncopeltus fasciatus [32,34]. In this study, the target gene expression level decreased significantly following dsRNA injection (Figure S4). In addition, the phenotype was consistent in the individuals of each RNAi group and could persist until the nymphs died or molted to the next instars. These results make RNAi an effective approach for studying the functions of these three melanin genes in this assassin bug.

The pigmentation process is initiated by $T H$, which hydroxylates tyrosine to DOPA. Our results showed that knockdown of $T H$ caused a complete loss of black pigment, with reduced cuticle sclerotization of the compound eyes, the legs, and the wing discs (Figure 2E-H). These results are consistent with those found in Tribolium, where downregulation of $T H$ led to decreased cuticle hardness and the loss of brown pigment [16]. These results confirm that pigments not only provide visual effects, but also serve as structural components of the cuticle $[35,36]$. Some dark brown markings were also observed in the dsTH nymphs (Figure 2H). A similar phenotype has been reported in Tribolium [16]. This may be explained as follows: Due to the loss of $T H$ function, tyrosine is excessively accumulated and then converted to the melanin precursor in the cuticle, and finally melanin is synthesized. Results 
of quantitative real-time PCR showed that $T H$ expression level was significantly lower in the first-instar nymphs than in others (Figure 1A). This observation may be explained by the fact that the first-instar nymph has a lower degree of cuticular sclerotization and less black pigment on its body compared with the other instars. Similarly, the level of TH expression correlated strongly with the intensity of the black markings in Papilio xuthus [19]. The expression level of TH was very high just after molting and then gradually decreased during the pigmentation process (Figure 1B). This indicates that $T H$ functions mainly at the beginning of the pigmentation. In addition, the TH expression level in adult body regions with black and white colors was much higher than that of the nymphs (Figure 1C). These results are consistent with previous studies in Tribolium and Papilio $[16,19]$. Taken together, our findings suggest that the black pigment in Platymeris is solely generated from the melanin pathway. $\mathrm{TH}$ functions not only in sclerotization, but also in the formation and the degree of pigmentation. It is noteworthy that downregulation of $\mathrm{TH}$ did not affect the formation of yellow pigment (Figure 2E). A similar phenotype was found in O. fasciatus, in which the orange color persisted in the individuals subjected to TH RNAi [29]. This finding suggests that the yellow pigment in hemimetabolous insects is not generated by the melanin pathway, but is synthesized by other pigmentation pathways.

\subsection{Brown Pigment Could Be Further Converted to Black Melanin}

After tyrosine is hydroxylated to produce DOPA, DOPA is then decarboxylated to form dopamine, which can be converted to NADA and NBAD. These substrates are secreted into the cuticle to form different colors. It is a controversial issue whether DOPA or dopamine is the main precursor of black melanin. Previous studies in Drosophila indicated that dopamine melanin was the main component of black pigment $[7,37]$. However, some other analyses provided evidence suggesting that DOPA melanin can contribute equally to black melanin $[3,5,6,8,9,38]$. In our study, downregulation of yellow led to the disappearance of black pigment, while the brown pigment was retained (Figure 3E). The freshly molted assassin bug was bright red, became brown gradually, and then the body turned black after the pigmentation progress was completed (Figures S1 and S2). Furthermore, yellow was highly expressed at the brown (five hours after molting) and dark brown stages (ten hours after molting), rather than at the very beginning of the pigmentation progress (Figure 1E). In addition, yellow RNAi also caused the brown phenotype in Oncopeltus [30]. Taken together, our results demonstrate that dopamine melanin makes a major contribution to black pigment in this assassin bug; however, it is firstly converted to brown pigment, and then the brown pigment is further converted to the black pigment.

In insects, yellow is a rapidly evolving gene family, which generates functionally diverse paralogs. In Drosophila, yellow-y was required to produce black pigment $[8,9,39]$. A similar function of yellow-y was reported in the lepidopteran insects, P. xuthus [40] and B. mori [41]. In Tribolium, yellow-y RNAi caused the loss of the pterostigma on the hindwing, whereas no effect was found on pigmentation of the body wall or elytron [14]. yellow-e was first studied in B. mori, indicating its requirement for the normal pattern of larval body color [42]. Unlike Bombyx, yellow-e RNAi in Tribolium did not affect color formation but caused dehydration-induced mortality in adults [17]. The expression level of yellow-y in Tribolium was the highest on pupal day three and declined substantially by the time of adult apolysis, while yellow-e expression occurred on pupal day three and peaked on pupal day four. In our study, the expression level of yellow gradually increased from the first- to fifth-instar nymphs and declined dramatically when the nymphs molted to adults (Figure 1D). This indicates that yellow may be essential for normal development in this species. RNAi of yellow indeed resulted in high mortality caused by cuticle degradation. Our results indicate that the yellow gene in this assassin bug seems to combine the functions of yellow-y and yellow-e. This yellow gene plays an essential role in both pigmentation and cuticle formation.

\section{3. aaNAT Suppresses the Formation of Black Melanin to Generate Colorless Sclerotin}

Finally, we analyzed the NADA branch, which suppresses melanin formation. aaNAT functions in this branch, where it can transform dopamine to NADA, after which the colorless sclerotin 
can be generated $[5-7,43,44]$. Early findings suggested that $a a N A T$ was mainly responsible for sclerotization [45-47]. It was first demonstrated that aaNAT was essential for insect color patterns in Bombyx $[25,26]$. In Tribolium, aaNAT was not required for larval, pupal, or pharate adult cuticle pigmentation, whereas aaNAT RNAi caused a significantly darker body color in adults [28]. However, Oncopeltus seemed to recruit aaNAT only in the hindwing, as aaNAT RNAi showed no effects on color patterns in the head, thorax, or abdomen [30]. Our aaNAT RNAi results were consistent with that of Bombyx, with a systemic black phenotype observed from the nymphs to the adults (Figures 4 and 5). We noticed that color of the sternum in the fifth instar nymphs was not apparently changed (Figure 4J), this is because this region has a low degree of sclerotization, while there is no such region in adults. The white spot of the hemelytra and the fenestrate area of the hindwing were invaded by black pigment in dsaaNAT-treated individuals (Figure 5C,F). The expression level of aaNAT was highest in adults, especially in the white spot of the hemelytra (Figure 1G,I). Furthermore, yellow was also highly expressed in the white spot (Figure 1F). These results support the "eraser" model proposed by Liu et al. [30]. This model proposes that in the fully black background, aaNAT is used for generating the colorless patches by "erasing" the preliminary melanin. Interestingly, aaNAT RNAi in this assassin bug led not only to the loss of white pigment, but also to the disappearance of yellow and red color patterns (Figure 5G-L). To investigate whether this phenotype was related to changes in the expression levels of $\mathrm{TH}$ and yellow, we performed quantitative real-time PCR with the aaNAT RNAi groups. We found that the expression level of $T H$ was not significantly different between dsaaNAT-treated nymphs and dsGFP-treated nymphs (Figure S5A). However, the expression level of yellow was significantly higher in the aaNAT RNAi group than that in the GFP RNAi group (Figure S5B). We speculate that aaNAT could inhibit the expression of yellow. When aaNAT is downregulated, the high expression of yellow can convert the accumulated dopamine to excessive black pigment, and this may be the reason for the global black phenotype in aaNAT RNAi groups.

\subsection{A Modified Melanin Pathway Suitable for P. biguttatus}

In summary, we provide evidence of a modified melanin pathway that is P. biguttatus specific (Figure 6). In this assassin bug, black melanin is solely generated by the melanin pathway. We propose that dopamine melanin is the main precursor of black pigment; however, yellow functions in the process of converting brown pigment to black pigment. The yellow pigment of this species is not synthesized by the melanin pathway, but by other pigmentation pathways. In the NADA branch, aaNAT acts as an "eraser" to maintain the white sclerotin on the preliminary black background. Furthermore, aaNAT can universally suppress the formation of black melanin by inhibiting yellow expression.

In this study, we showed that this assassin bug is an excellent model for studying insect pigmentation. We also demonstrated a fascinating phenotype of aaNAT, which is completely different from that of Oncopeltus. However, it remains unclear why gene functions in this species are more similar to that of holometabolous insects (such as Tribolium and Bombyx), rather than hemimetabolous insects (such as Oncopeltus). 


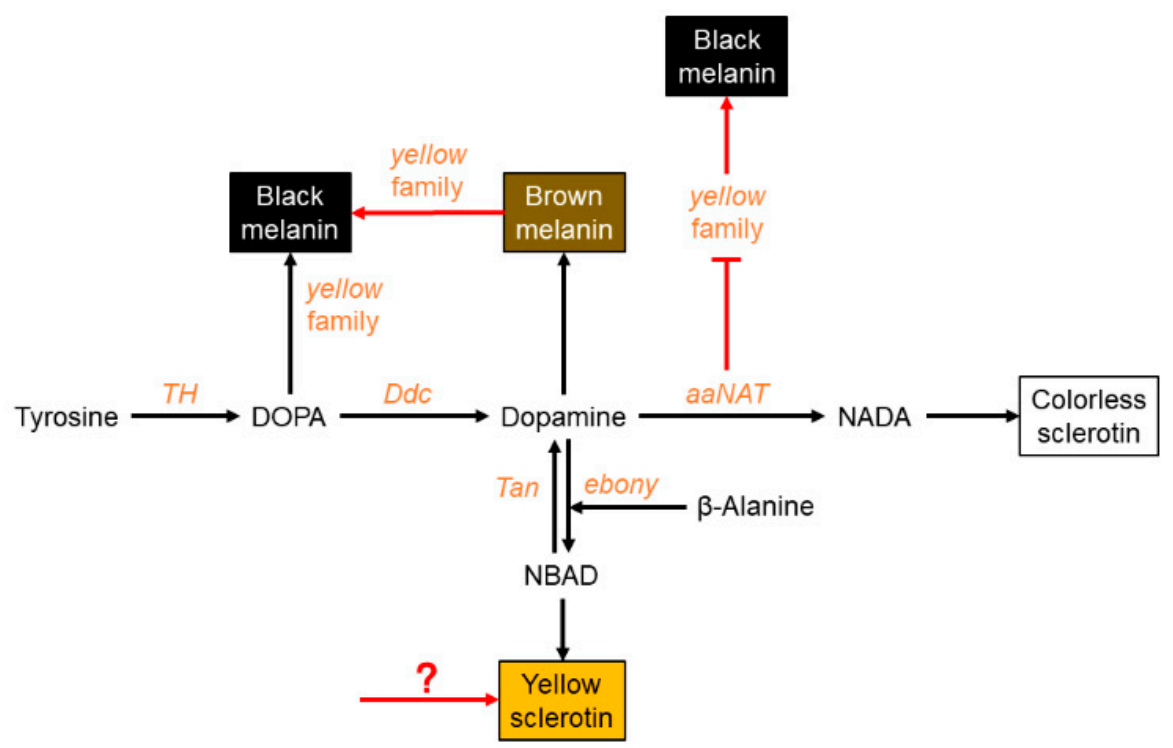

Figure 6. A proposed melanin pathway in P. biguttatus. In this assassin bug, black melanin is solely generated from the melanin pathway, and dopamine melanin is the main precursor of black pigment. However, yellow functions in the process of converting brown melanin to black melanin (red arrow). The yellow pigment of this species is not synthesized by the NBAD branch, but by other pigmentation pathways. In the NADA branch, aaNAT acts as an "eraser" to maintain the white spot on the preliminary black background. Furthermore, aaNAT can inhibit yellow expression, thereby inhibiting the formation of black melanin (red suppression symbol).

\section{Materials and Methods}

\subsection{Insects}

P. biguttatus was reared in the lab for five years. These insects were fed once a week with two species of live larvae: Mealworms, Tenebrio molitor, and greater wax moth, Galleria mellonella. They were reared under a photoperiod of $16 \mathrm{~h} / 8 \mathrm{~h} \mathrm{light/dark,} \mathrm{at} 27^{\circ} \mathrm{C}$ and $60 \%$ relative humidity during the daytime and at $25^{\circ} \mathrm{C}$ and $65 \%$ relative humidity at night.

\subsection{Total RNA Extraction and $c D N A$ Synthesis}

The yellow or black regions of the legs were dissected from one fourth-instar nymph, one fifth-instar nymph, and one adult, respectively. The white or black regions of the hemelytra were dissected from five adults. Total RNA of other groups was extracted from one whole insect, except for the first-instar nymph $(n=2)$. Total RNA of each group with three biological replicates was extracted using the RNeasy Mini Kit (Qiagen, Düsseldorf, Germany) following the manufacturer's instructions. Concentrations of total RNA were measured using a NanoDrop 2000 (Thermo Fisher Scientific, Waltham, MA, USA), and the ratio of A260/A280 (absorbance at $260 \mathrm{~nm} /$ absorbance at 280 $\mathrm{nm}$ ) for all RNA samples was between 1.8 and 2.0. RNA integrity was determined using agarose gel electrophoresis. RNA was considered intact by the presence of a clear 18S rRNA band [48]. All RNA was then treated with DNase I using the DNase Max Kit (Qiagen, Düsseldorf, Germany) following the manufacturer's instructions. Complementary DNA (cDNA) was synthesized from a standard amount of total RNA $(1.9 \mu \mathrm{g})$ using the GoScript Reverse Transcription System (Promega, Madison, WI, USA). RNA was incubated with Oligo $(\mathrm{dT})_{15}$ Primer $(0.5 \mu \mathrm{g})$ and Random Primers $(0.5 \mu \mathrm{g})$ at $70{ }^{\circ} \mathrm{C}$ for $5 \mathrm{~min}$. Each reaction included GoScript Reverse Transcriptase, Recombinant RNasin Ribonuclease Inhibitor, and $\mathrm{MgCl}_{2}$. Then incubated at $25^{\circ} \mathrm{C}$ for $5 \mathrm{~min}$ (anneal), $42{ }^{\circ} \mathrm{C}$ for $1 \mathrm{~h}$ (extend). Before proceeding with qPCR, inactivated the reverse transcriptase at $70^{\circ} \mathrm{C}$ for $15 \mathrm{~min}$. 
Sequences of $T H$, yellow, and aaNAT were identified from the transcriptome data of this assassin bug by performing BLAST searches (tblastn) using BioEdit 7.2.5 software (Ibis Biosciences, Carlsbad, CA, USA). These sequences were deposited in the GenBank: MK426755 (TH), MK426756 (yellow), and MK426757 (aaNAT). The sequences of these three genes are given in Table S1, and their orthologs were confirmed by molecular phylogenetic analysis (Figure S3).

\subsection{RNA Interference}

dsRNAs targeting the conserved regions of $T H$, yellow, and aaNAT were synthesized using the T7 RiboMAX Express RNAi System Kit (Promega, Madison, WI, USA) from the corresponding T7-DNA. T7-DNA was synthesized using pairs of primers containing T7 promoter sequences (TAA TAC GAC TCA CTA TAG G) at the $5^{\prime}$ ends [28]. For the primers used to amplify these target regions, see Table S2. The lengths of the dsRNA products were $483 \mathrm{bp}$ for $\mathrm{TH}$ (nucleotides 236-718), $448 \mathrm{bp}$ for yellow (nucleotides 461-908), and $447 \mathrm{bp}$ for aaNAT (nucleotides 118-564). dsRNA for green fluorescent protein (GFP) (GenBank number XM_013480425.1, nucleotides 157-576) was used as a control. Primer set for dsGFP was 5' -(T7)-CACAAGTTCAGCGTGTCCG-3' and 5'-(T7)-GTTCACCTTGATGCCGTTC-3' .

The prepared dsRNA was then injected into the abdomen of the early fourth-instar nymphs, using a Hamilton syringe with a 33-gauge needle [49]. A total of $20 \mu \mathrm{g}$ (approximately $5 \mu \mathrm{L}$ ) dsRNA was injected into each individual. An equivalent amount of dsGFP was injected into the control nymphs at the same developmental stage.

\subsection{Quantitative Real-Time PCR}

To determine the efficiency of the RNAi approach and the expression profiles of $T H$, yellow, and $a a N A T$, we performed quantitative real-time PCR analyses. The cDNA templates were prepared from total RNA isolated from whole nymphs at $48 \mathrm{~h}$ post injection $(n=3)$. Total RNA was independently extracted for each of the three replicates. The sequences of the gene-specific quantitative real-time PCR primers are presented in Table S2. Quantitative real-time PCR was conducted using TB Green Premix Ex Taq II (Tli RNaseH Plus) (Takara, Shiga, Japan) and the CFX96 Real-Time PCR Detection System (Bio-Rad, Portland, ME, USA). The PCR mixtures were contained in the Axygen $0.2 \mathrm{~mL}$ thin-wall 8 strip PCR tubes (Corning, Corning, NY, USA), included $2 \mu \mathrm{L}$ of the synthesized cDNA, $0.5 \mu \mathrm{L}$ of each primer $(10 \mu \mathrm{M}), 12.5 \mu \mathrm{L}$ of TB Green Premix Ex Taq II (Tli RNaseH Plus), and $9.5 \mu \mathrm{L}$ of $\mathrm{ddH}_{2} \mathrm{O}$. Each experiment was performed with three technical replicates and biological replicates. The thermocycling program was as followed: Initial denaturation at $95^{\circ} \mathrm{C}$ for $30 \mathrm{~s}, 40$ cycles of $95^{\circ} \mathrm{C}$ for $5 \mathrm{~s}$ and $60^{\circ} \mathrm{C}$ for $30 \mathrm{~s}$, followed by $95^{\circ} \mathrm{C}$ for $10 \mathrm{~s}$, and from 65 to $95^{\circ} \mathrm{C}$ in increments of $0.5^{\circ} \mathrm{C}$ for $5 \mathrm{~s}$ [28]. The elongation factor 1 alpha (EF1 $\alpha$ ) (GenBank number MK744598) was used as a reference gene. The primers for quantification of $E F 1 \alpha$ expression were $5^{\prime}$-CATCTTTGTTGAGTTTGTCG- $3^{\prime}$ and $5^{\prime}$-GACCTGTAGTTGTAGATTTACC- $3^{\prime}$. At the end of each quantitative real-time PCR run, a melting curve was generated to confirm the presence of a single peak and rule out the possibility of primer dimer and non-specific product formation. The quantification cycle $(\mathrm{Cq})$ was determined and was used for comparative quantitative analysis. The $2^{-\Delta \Delta \mathrm{Ct}}$ method was used to calculate the relative expression levels of the target genes in all groups [50]. Primer amplification efficiencies, correlation coefficient $\left(r^{2}\right)$, slope, and $y$-intercept were presented in Table S3. No template controls (NTC) were performed with every experiment to determine if PCR mixtures were contaminated or unintended products were amplified, such as the primer dimers [51,52]. No Cqs were detected in all NTC experiments. The column chart was drawn using GraphPad Prism 7.0 software (GraphPad, San Diego, CA, USA). SPSS Statistics 25.0 software (IBM, Armonk, NY, USA) was used to perform unpaired Student's $t$ test (for two groups) and one-way ANOVA analysis (for more than two groups). 


\subsection{Image Processing}

Images were taken using an SZX7 stereoscope (Olympus, Tokyo, Japan) and a 7D camera (Canon, Tokyo, Japan). The images under a particular magnification were taken under the same light, exposure time, aperture, and white balance conditions.

Supplementary Materials: The following are available online at http://www.mdpi.com/1422-0067/20/11/2728/s1. Figure S1. Pigmentation process of the fifth-instar nymph. (A) The newly molted fifth-instar nymph was bright red, with a yellow annulation on the leg; (B) over time, the red regions gradually turned brown. (C) The fifth/instar nymph was completely pigmented after about $18 \mathrm{~h}$. (D) The abdomen changed from black to brownish red after feeding. V, fifth/instar nymph; h, hours after molting; AF, after feeding. Scale bar, $5 \mathrm{~mm}$. Figure S2. Pigmentation process of the adult. Similar to the fifth/instar nymph, the future black regions of the adult (D) were first bright red (A), then turned brown (B), and then turned dark brown (C). The adult was completely pigmented after about 48 $\mathrm{h}$. The white spot of the hemelytra remained colorless during the pigmentation process. The region of the dorsal abdomen covered by the forewings was red (E). The membranous hindwing was light brown with a fenestrate area (F, the black arrowhead). A, adult; h, hours after molting. Scale bars, $5 \mathrm{~mm}$ (D), $2.5 \mathrm{~mm}(\mathrm{E}, \mathrm{F})$. Figure S3. Phylogenetic analysis of TH, yellow, and aaNAT based on their deduced amino acid sequences. The molecular phylogenetic tree was constructed by MEGA7 using the neighbor-joining method [53]. Branch support values are bootstrap percentages from 5000 replicates. The scale bars indicate the estimated number of amino acid substitutions per site. Boxes indicate the P. biguttatus orthologs. The accession numbers of these sequences are in the parentheses. Figure S4. RNAi efficiency of $T H$, yellow, and aaNAT. The expression levels of TH (A), yellow (B), and $a a N A T(\mathrm{C})$ were significantly decreased in dsRNA-treated nymphs compared with dsGFP-treated nymphs. Data are shown as the mean values $\pm \mathrm{SE}$ (error bars) $(n=3) .{ }^{*} p<0.05,{ }^{* *} p<0.01,{ }^{* * *} p<0.001$, according to the Student's $t$ test. Figure S5. Expression levels of TH and yellow in dsaaNAT-treated nymphs and dsGFP-treated nymphs. The expression level of $T H$ was not significantly different between dsaaNAT-treated nymphs and dsGFP-treated nymphs (A). However, the expression level of yellow was significantly higher in the aaNAT RNAi group than in the GFP RNAi group (B). Data are shown as the mean values \pm SE (error bars) $(n=3)$. ${ }^{*} p<0.05$, ${ }^{* *} p<0.01,{ }^{* * *} p<0.001$, according to the Student's $t$ test.

Author Contributions: Formal analysis, Y.Z., H.L., J.D., and J.Z.; project administration, W.C. and J.S.; resources, Y.Z.; supervision, W.C. and J.S.; writing—original draft, Y.Z. and H.L.; writing—review and editing, Y.Z., W.C., J.S., and H.L.

Funding: This research was financially supported by two National Natural Science Foundation of China (Nos. 31730086, 31872295).

Conflicts of Interest: The authors declare no conflict of interest.

$\begin{array}{ll}\text { Abbreviations } \\ \text { aaNAT } & \text { arylalkylamine- } N \text {-acetyltransferase } \\ \mathrm{bp}(\mathrm{s}) & \text { base pair(s) } \\ \mathrm{Cq} & \text { quantification cycle } \\ \text { Ddc } & \text { DOPA decarboxylase } \\ \text { DOPA } & \text { dihydroxyphenylalanine } \\ \text { dsRNA } & \text { double-stranded RNA } \\ \text { EF1 } \alpha & \text { elongation factor } 1 \text { alpha } \\ \text { GFP } & \text { green fluorescent protein } \\ \text { NADA } & \text { N-acetyl dopamine } \\ \text { NBAD } & N \text { - } \beta \text {-alanyl dopamine } \\ \text { NTC } & \text { no template controls } \\ \text { qPCR } & \text { quantitative real-time PCR } \\ \text { RNAi } & \text { RNA interference } \\ \text { TH } & \text { tyrosine hydroxylase }\end{array}$

\section{References}

1. True, J.R. Insect melanism: The molecules matter. Trends Ecol. Evol. 2003, 18, 640-647. [CrossRef]

2. Wittkopp, P.J.; Beldade, P. Development and evolution of insect pigmentation: Genetic mechanisms and the potential consequences of pleiotropy. Semin. Cell Dev. Biol. 2009, 20, 65-71. [CrossRef]

3. Nijhout, H.F. The Development and Evolution of Butterfly Wing Patterns; Smithsonian Institution Press: Washington, DC, USA, 1991; p. 297. 
4. Maranda, B.; Hodgetts, R. A characterization of dopamine acetyltransferase in Drosophila melanogaster. Insect Biochem. 1977, 7, 33-43. [CrossRef]

5. Wittkopp, P.J.; Carroll, S.B.; Kopp, A. Evolution in black and white: Genetic control of pigment patterns in Drosophila. Trends Genet. 2003, 19, 495-504. [CrossRef]

6. Wittkopp, P.J.; Williams, B.L.; Selegue, J.E.; Carroll, S.B. Drosophila pigmentation evolution: Divergent genotypes underlying convergent phenotypes. Proc. Natl. Acad. Sci. USA 2003, 100, 1808-1813. [CrossRef]

7. Wright, T.R. The genetics of biogenic amine metabolism, sclerotization, and melanization in Drosophila melanogaster. Adv. Genet. 1987, 24, 127-222. [CrossRef]

8. Wittkopp, P.J.; True, J.R.; Carroll, S.B. Reciprocal functions of the Drosophila yellow and ebony proteins in the development and evolution of pigment patterns. Development 2002, 129, 1849-1858.

9. Wittkopp, P.J.; Vaccaro, K.; Carroll, S.B. Evolution of yellow gene regulation and pigmentation in Drosophila. Curr. Biol. 2002, 12, 1547-1556. [CrossRef]

10. True, J.R.; Yeh, S.D.; Hovemann, B.T.; Kemme, T.; Meinertzhagen, I.A.; Edwards, T.N.; Liou, S.R.; Han, Q.; Li, J. Drosophila tan encodes a novel hydrolase required in pigmentation and vision. PLoS Genet. 2005, 1, e63. [CrossRef]

11. Jeong, S.; Rebeiz, M.; Andolfatto, P.; Werner, T.; True, J.; Carroll, S.B. The evolution of gene regulation underlies a morphological difference between two Drosophila sister species. Cell 2008, 132, 783-793. [CrossRef]

12. Wittkopp, P.J.; Stewart, E.E.; Arnold, L.L.; Neidert, A.H.; Haerum, B.K.; Thompson, E.M.; Akhras, S.; Smith-Winberry, G.; Shefner, L. Intraspecific polymorphism to interspecific divergence: Genetics of pigmentation in Drosophila. Science 2009, 326, 540-544. [CrossRef]

13. Arakane, Y.; Lomakin, J.; Beeman, R.W.; Muthukrishnan, S.; Gehrke, S.H.; Kanost, M.R.; Kramer, K.J. Molecular and functional analyses of amino acid decarboxylases involved in cuticle tanning in Tribolium castaneum. J. Biol. Chem. 2009, 284, 16584-16594. [CrossRef]

14. Arakane, Y.; Dittmer, N.T.; Tomoyasu, Y.; Kramer, K.J.; Muthukrishnan, S.; Beeman, R.W.; Kanost, M.R. Identification, mRNA expression and functional analysis of several yellow family genes in Tribolium castaneum. Insect Biochem. Mol. Biol. 2010, 40, 259-266. [CrossRef]

15. Tomoyasu, Y.; Arakane, Y.; Kramer, K.J.; Denell, R.E. Repeated co-options of exoskeleton formation during wing-to-elytron evolution in beetles. Curr. Biol. 2009, 19, 2057-2065. [CrossRef]

16. Gorman, M.J.; Arakane, Y. Tyrosine hydroxylase is required for cuticle sclerotization and pigmentation in Tribolium castaneum. Insect Biochem. Mol. Biol. 2010, 40, 267-273. [CrossRef]

17. Noh, M.Y.; Kramer, K.J.; Muthukrishnan, S.; Beeman, R.W.; Kanost, M.R.; Arakane, Y. Loss of function of the yellow-e gene causes dehydration-induced mortality of adult Tribolium castaneum. Dev. Biol. 2015, 399, 315-324. [CrossRef]

18. Koch, P.B.; Behnecke, B. The molecular basis of melanism and mimicry in a swallowtail butterfly. Curr. Biol. 2000, 10, 591-594. [CrossRef]

19. Futahashi, R.; Fujiwara, H. Melanin-synthesis enzymes coregulate stage-specific larval cuticular markings in the swallowtail butterfly, Papilio xuthus. Dev. Genes Evol. 2005, 215, 519-529. [CrossRef]

20. Niu, B.L.; Shen, W.F.; Liu, Y.; Weng, H.B.; He, L.H.; Mu, J.J.; Wu, Z.L.; Jiang, P.; Tao, Y.Z.; Meng, Z.Q. Cloning and RNAi-mediated functional characterization of MaLac2 of the pine sawyer, Monochamus alternatus. Insect Mol. Biol. 2008, 17, 303-312. [CrossRef]

21. Futahashi, R.; Banno, Y.; Fujiwara, H. Caterpillar color patterns are determined by a two-phase melanin gene prepatterning process: New evidence from tan and laccase2. Evol. Dev. 2010, 12, 157-167. [CrossRef]

22. Elias-Neto, M.; Soares, M.P.; Simoes, Z.L.; Hartfelder, K.; Bitondi, M.M. Developmental characterization, function and regulation of a Laccase 2 encoding gene in the honey bee, Apis mellifera (Hymenoptera, Apinae). Insect Biochem. Mol. Biol. 2010, 40, 241-251. [CrossRef]

23. Ferguson, L.C.; Maroja, L.; Jiggins, C.D. Convergent, modular expression of ebony and tan in the mimetic wing patterns of Heliconius butterflies. Dev. Genes Evol. 2011, 221, 297-308. [CrossRef]

24. Mehere, P.; Han, Q.; Christensen, B.M.; Li, J. Identification and characterization of two arylalkylamine $\mathrm{N}$-acetyltransferases in the yellow fever mosquito, Aedes aegypti. Insect Biochem. Mol. Biol. 2011, 41, 707-714. [CrossRef]

25. Dai, F.Y.; Qiao, L.; Tong, X.L.; Cao, C.; Chen, P.; Chen, J.; Lu, C.; Xiang, Z.H. Mutations of an arylalkylamine $N$-acetyltransferase, Bm-iAANAT, are responsible for silkworm melanism mutant. J. Biol. Chem. 2010, 285, 19553-19560. [CrossRef] 
26. Zhan, S.; Guo, Q.; Li, M.; Li, M.; Li, J.; Miao, X.; Huang, Y. Disruption of an N-acetyltransferase gene in the silkworm reveals a novel role in pigmentation. Development 2010, 137, 4083-4090. [CrossRef]

27. Osanai-Futahashi, M.; Ohde, T.; Hirata, J.; Uchino, K.; Futahashi, R.; Tamura, T.; Niimi, T.; Sezutsu, H. A visible dominant marker for insect transgenesis. Nat. Commun. 2012, 3, 1295. [CrossRef]

28. Noh, M.Y.; Koo, B.; Kramer, K.J.; Muthukrishnan, S.; Arakane, Y. Arylalkylamine N-acetyltransferase 1 gene (TcAANAT1) is required for cuticle morphology and pigmentation of the adult red flour beetle, Tribolium castaneum. Insect Biochem. Mol. Biol. 2016, 79, 119-129. [CrossRef]

29. Liu, J.; Lemonds, T.R.; Popadic, A. The genetic control of aposematic black pigmentation in hemimetabolous insects: Insights from Oncopeltus fasciatus. Evol. Dev. 2014, 16, 270-277. [CrossRef]

30. Liu, J.; Lemonds, T.R.; Marden, J.H.; Popadic, A. A pathway analysis of melanin patterning in a Hemimetabolous insect. Genetics 2016, 203, 403-413. [CrossRef]

31. Lemonds, T.R.; Liu, J.; Popadić, A. The contribution of the melanin pathway to overall body pigmentation during ontogenesis of Periplaneta americana. Insect Sci. 2016, 23, 513-519. [CrossRef]

32. Singh, I.K.; Singh, S.; Mogilicherla, K.; Shukla, J.N.; Palli, S.R. Comparative analysis of double-stranded RNA degradation and processing in insects. Sci. Rep. 2017, 7, 17059. [CrossRef]

33. Li, Z.; Zeng, B.; Ling, L.; Xu, J.; You, L.; Aslam, A.F.; Tan, A.; Huang, Y. Enhancement of larval RNAi efficiency by over-expressing Argonaute2 in Bombyx mori. Int. J. Biol. Sci. 2015, 11, 176. [CrossRef]

34. Angelini, D.R.; Kaufman, T.C. Functional analyses in the milkweed bug Oncopeltus fasciatus (Hemiptera) support a role for Wnt signaling in body segmentation but not appendage development. Dev. Biol. 2005, 283, 409-423. [CrossRef]

35. Moussian, B. Recent advances in understanding mechanisms of insect cuticle differentiation. Insect Biochem. Mol. Biol. 2010, 40, 363-375. [CrossRef]

36. Kronforst, M.R.; Barsh, G.S.; Kopp, A.; Mallet, J.; Monteiro, A.; Mullen, S.P.; Protas, M.; Rosenblum, E.B.; Schneider, C.J.; Hoekstra, H.E. Unraveling the thread of nature's tapestry: The genetics of diversity and convergence in animal pigmentation. Pigment. Cell Melanoma Res. 2012, 25, 411-433. [CrossRef]

37. Walter, M.F.; Black, B.C.; Afshar, G.; Kermabon, A.Y.; Wright, T.R.; Biessmann, H. Temporal and spatial expression of the yellow gene in correlation with cuticle formation and DOPA decarboxylase activity in Drosophila development. Dev. Biol. 1991, 147, 32-45. [CrossRef]

38. Johnson, J.; Li, J.; Christensen, B. Cloning and characterization of a dopachrome conversion enzyme from the yellow fever mosquito, Aedes aegypti. Insect Biochem. Mol. Biol. 2001, 31, 1125-1135. [CrossRef]

39. Han, Q.; Fang, J.M.; Ding, H.Z.; Johnson, J.K.; Christensen, B.M.; Li, J.Y. Identification of Drosophila melanogaster yellow-f and yellow-f2 proteins as dopachrome-conversion enzymes. Biochem. J. 2002, 368, 333-340. [CrossRef]

40. Futahashi, R.; Fujiwara, H. Regulation of 20-hydroxyecdysone on the larval pigmentation and the expression of melanin synthesis enzymes and yellow gene of the swallowtail butterfly, Papilio xuthus. Insect Biochem. Mol. Biol. 2007, 37, 855-864. [CrossRef]

41. Futahashi, R.; Sato, J.; Meng, Y.; Okamoto, S.; Daimon, T.; Yamamoto, K.; Suetsugu, Y.; Narukawa, J.; Takahashi, H.; Banno, Y.; et al. Yellow and ebony are the responsible genes for the larval color mutants of the silkworm Bombyx mori. Genetics 2008, 180, 1995-2005. [CrossRef]

42. Ito, K.; Katsuma, S.; Yamamoto, K.; Kadono-Okuda, K.; Mita, K.; Shimada, T. Yellow-e determines the color pattern of larval head and tail spots of the silkworm Bombyx mori. J. Biol. Chem. 2010, 285, 5624-5629. [CrossRef]

43. Hintermann, E.; Jenö, P.; Meyer, U.A. Isolation and characterization of an arylalkylamine N-acetyltransferase from Drosophila melanogaster. FEBS Lett. 1995, 375, 148-150. [CrossRef]

44. Brodbeck, D.; Amherd, R.; Callaerts, P.; Hintermann, E.; Meyer, U.A.; Affolter, M. Molecular and biochemical characterization of the aaNAT1 (Dat) locus in Drosophila melanogaster: Differential expression of two gene products. DNA Cell Biol. 1998, 17, 621-633. [CrossRef]

45. Walter, M.F.; Zeineh, L.L.; Black, B.C.; McIvor, W.E.; Wright, T.R.; Biessmann, H. Catecholamine metabolism and in vitro induction of premature cuticle melanization in wild type and pigmentation mutants of Drosophila melanogaster. Arch. Insect Biochem. Physiol. 1996, 31, 219-233. [CrossRef]

46. Andersen, S.O. Insect cuticular sclerotization: A review. Insect Biochem. Mol. Biol. 2010, 40, 166-178. [CrossRef] 
47. Gibert, J.M.; Peronnet, F.; Schlotterer, C. Phenotypic plasticity in Drosophila pigmentation caused by temperature sensitivity of a chromatin regulator network. PLoS Genet. 2007, 3, e30. [CrossRef]

48. Fujiwara, H.; Ishikawa, H. Molecular mechanism of introduction of the hidden break into the 28S rRNA of insects: Implication based on structural studies. Nucleic Acids Res. 1986, 14, 6393-6401. [CrossRef]

49. Chesebro, J.; Hrycaj, S.; Mahfooz, N.; Popadić, A. Diverging functions of Scr between embryonic and post-embryonic development in a hemimetabolous insect, Oncopeltus fasciatus. Dev. Biol. 2009, 329, 142-151. [CrossRef]

50. Livak, K.J.; Schmittgen, T.D. Analysis of relative gene expression data using real-time quantitative PCR and the 2- $\Delta \Delta$ CT method. Methods 2001, 25, 402-408. [CrossRef]

51. Bustin, S.A.; Benes, V.; Garson, J.A.; Hellemans, J.; Huggett, J.; Kubista, M.; Vandesompele, J. The MIQE guidelines: Minimum information for publication of quantitative real-time PCR experiments. Clin. Chem. 2009, 55, 611-622. [CrossRef]

52. Bustin, S.A.; Beaulieu, J.F.; Huggett, J.; Jaggi, R.; Kibenge, F.S.; Olsvik, P.A.; Toegel, S. MIQE précis: Practical implementation of minimum standard guidelines for fluorescence-based quantitative real-time PCR experiments. BMC Mol. Biol. 2010, 11, 74. [CrossRef]

53. Kumar, S.; Stecher, G.; Tamura, K. MEGA7: Molecular evolutionary genetics analysis version 7.0 for bigger datasets. Mol. Biol. Evol. 2016, 33, 1870-1874. [CrossRef]

(C) 2019 by the authors. Licensee MDPI, Basel, Switzerland. This article is an open access article distributed under the terms and conditions of the Creative Commons Attribution (CC BY) license (http://creativecommons.org/licenses/by/4.0/). 